\title{
Erratum to: National Survey on Excellence Centers and Reference Centers for Hypertension Diagnosis and Treatment: Geographical Distribution, Medical Facilities and Diagnostic Opportunities
}

\author{
Giuliano Tocci - Nicola De Luca $\cdot$ Riccardo Sarzani $\cdot$ Ettore Ambrosioni $\cdot$ Claudio Borghi \\ Santina Cottone - Cesare Cuspidi · Francesco Fallo • Claudio Ferri • Alberto Morganti • \\ Maria Lorenza Muiesan · Leonardo Sechi $\cdot$ Agostino Virdis · Giuseppe Mancia • Massimo Volpe
}

Published online: 24 June 2014

(c) Springer International Publishing Switzerland 2014

Erratum to: High Blood Press Cardiovasc Prev (2014)

21:29-36

DOI 10.1007/s40292-013-0034-z

Unfortunately, an error occured in Fig. 1. The corrected figure is given below.

The online version of the original article can be found under doi:10.1007/s40292-013-0034-z.

\section{G. Tocci $(\bowtie) \cdot$ M. Volpe}

Division of Cardiology, Department of Clinical and Molecular Medicine, Faculty of Medicine and Psychology, University of Rome "Sapienza", Sant'Andrea Hospital, Via di Grottarossa, 1035-39, 00189 Rome, Italy

e-mail: giuliano.tocci@uniroma1.it

M. Volpe

e-mail: massimo.volpe@uniroma1.it

\section{N. De Luca}

Department of Clinical and Experimental Medicine,

Universityof Napoli Federico II, Napoli, Italy

R. Sarzani

Division of Internal Medicine, University of Marche,

Ancona, Italy

E. Ambrosioni · C. Borghi

Division of Internal Medicine, University of Bologna,

Bologna, Italy

\section{S. Cottone}

Department of Internal Medicine and Specialties, University

of Palermo, Palermo, Italy

C. Cuspidi · G. Mancia

Department of Clinical Medicine and Prevention, University of Milano Bicocca, Centro Ricerche Cliniche, Istituto Auxologico Italiano IRCCS, Milano, Italy

F. Fallo

Department of Medicine, Clinica Medica 3, University of Padova, Padova, Italy

\section{Ferri}

Division of Internal Medicine 1, Hypertension and Cardiovascular Prevention Unit, School of Internal Medicine, University of L'Aquila, San Salvatore Hospital, L'Aquila, Italy

\section{A. Morganti}

Division of Internal Medicine, Hypertension Unit, San Giuseppe

Hospital, Milano, Italy 


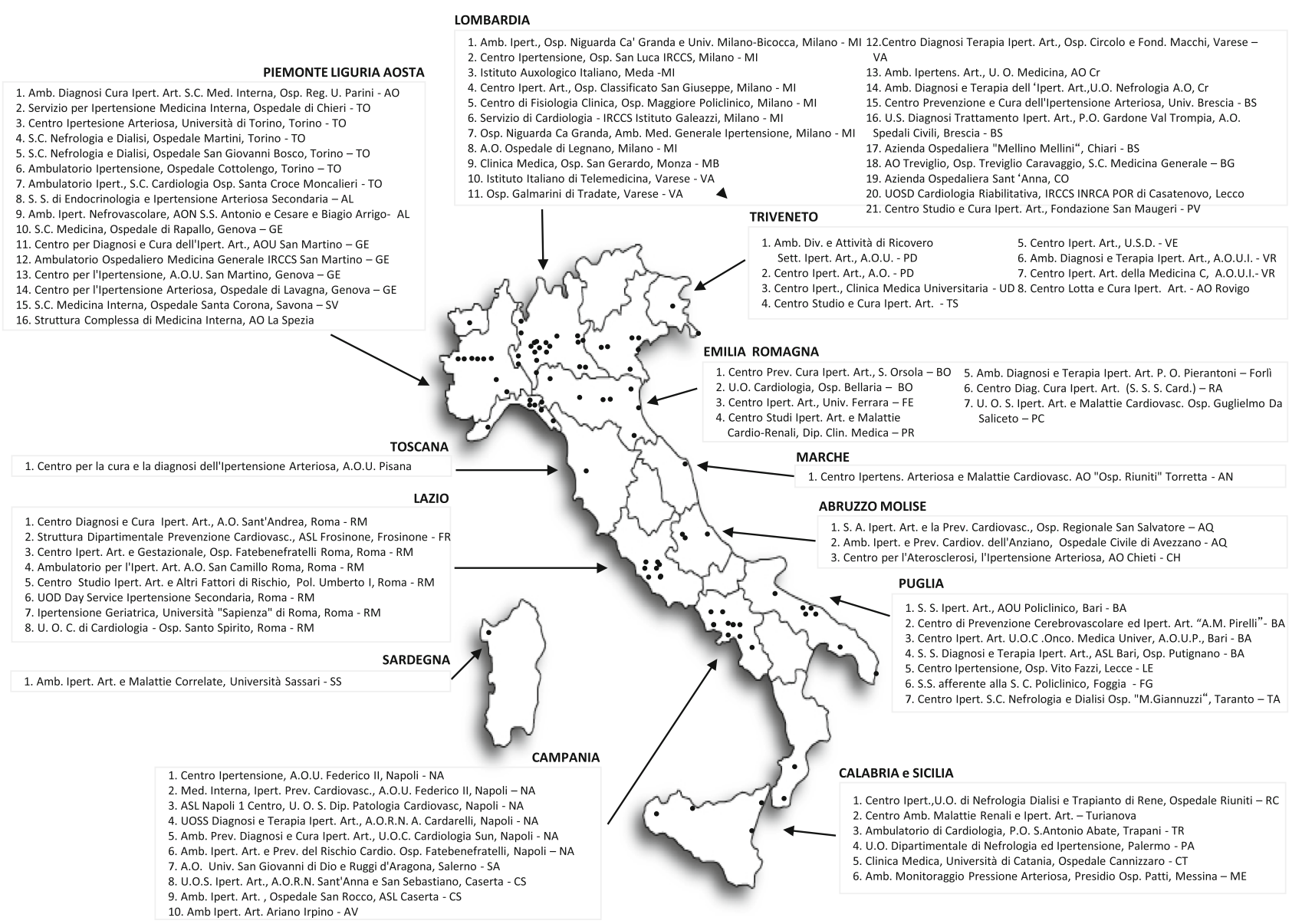

Fig. 1 Distribution of excellence centers and reference centers for the diagnosis and treatment of hypertension in Italy

\section{L. Muiesan}

Clinical Medicine, Department of Medical and Surgical Sciences, University of Brescia, Brescia, Italy

\section{L. Muiesan}

Department of Medical and Surgical Sciences, University of Brescia, Brescia, Italy

L. Sechi

Clinica Medica, Department of Clinical and Experimental Medicine, University of Udine, Udine, Italy

A. Virdis

Department of Internal Medicine, University of Pisa, Pisa, Italy 